\title{
Formation and Evolution of Monoepoxy Fatty Acids in Thermoxidized Olive and Sunflower Oils and Quantitation in Used Frying Oils from Restaurants and Fried-Food Outlets
}

\author{
Joaquín Velasco, Susana Marmesat, Olivier Bordeaux, \\ Gloria Márquez-Ruiz, and CARmen Dobarganes*
}

Instituto de la Grasa (CSIC), Avenida Padre García Tejero 4, 41012 Sevilla, Spain

\begin{abstract}
The formation and evolution of monoepoxy fatty acids, arising from oleic and linoleic acids, were investigated in olive oil and conventional sunflower oil, representatives of monounsaturated and polyunsaturated oils, respectively, during thermoxidation at $180^{\circ} \mathrm{C}$ for 5,10 , and $15 \mathrm{~h}$. Six monoepoxy fatty acids, cis-9,10- and trans-9,10-epoxystearate, arising from oleic acid, and cis-9,10-, trans-9,10-, cis-12,13-, and trans-12,13-epoxyoleate, arising from linoleic acid, were analyzed by gas chromatography after oil derivatization to fatty acid methyl esters. Considerable amounts, ranging from 4.29 to $14.24 \mathrm{mg} / \mathrm{g}$ of oil in olive oil and from 5.10 to $9.44 \mathrm{mg} / \mathrm{g}$ of oil in sunflower oil, were found after the heating periods assayed. Results showed that the monoepoxides quantitated constituted a major group among the oxidized fatty acid monomers formed at high temperature. For similar levels of degradation, higher contents of the monoepoxides were found in olive oil than in sunflower oil. Ten used frying oils from restaurants and fried-food outlets in Spain were analyzed to determine the contents of the monoepoxides in real frying oil samples. Levels ranged from 3.37 to $14.42 \mathrm{mg} / \mathrm{g}$ of oil. Results show that, for similar degradation levels, the monoepoxides were more abundant in the monounsaturated oils than in the polyunsaturated oils.
\end{abstract}

KEYWORDS: Epoxides; monoepoxy fatty acid methyl esters; olive oil; sunflower oil; thermoxidation; used frying oils

\section{INTRODUCTION}

Lipid oxidation is the cause of important deteriorative changes in the chemical, sensory, and nutritional properties of foods. In particular, the question of whether oxidized fats in the diet may be detrimental to health is nowadays of the utmost concern $(1$, 2).

Oxidation compounds in foods are primarily formed during processing, including every step from the raw product selection to the final preparation before consumption. During oxidation at low temperature not more than $4-5 \%$ of triacylglycerols (TAGs) are expected to be oxidized in the ingested foods. At this extent of oxidation, which makes up $\sim 100$ mequiv of $\mathrm{O}_{2} /$ $\mathrm{kg}$ of fat, rancidity is easily recognizable and hence the oil or food is rejected by the consumer (3). In contrast, in the case of used frying fats the present official regulations in many European countries establish the limit of new compounds formed in them as high as $25 \%$ (4), but even this level is often considerably surpassed in a significant number of oils and fats in fast-food outlets (5). Therefore, most of the oxidized fats in foods are expected to come from fats and oils heated at high temperature and, more specifically, from frying fats.

Analytical techniques for the evaluation of heated or used frying fats are essential to clarify the structures and the amounts of oxidation compounds that might impair the nutritional value of food lipids. The main groups of new compounds formed, that is, TAG polymers, TAG dimers, oxidized TAG monomers, diacylglycerols, and free fatty acids, can be quantitated by combination of adsorption and size exclusion chromatography (6). Among these groups, quantitation of oxidized TAG monomers seems to be of top priority, since they constitute the most abundant group of oxidation compounds in foods and, moreover, have consistently shown high overall absorption ( 7 , 8). Oxidized TAG monomers comprise TAG molecules bearing different oxygen-containing functional groups such as hydroperoxide, aldehyde, ketone, hydroxide, and epoxide (9). Specifically, absorption of dietary labeled epoxy fatty acids incorporated in TAGs has been recently reported in humans. This finding has added prominence to the need of quantitating the human consumption of dietary oxidation products (10).

In a previous study, formation of monoepoxides in considerable amounts was demonstrated in model systems of triolein and trilinolein heated at frying temperature. Thermoxidized triolein and trilinolein were derivatized to fatty acid methyl esters (FAMEs), and monoepoxy FAMEs were analyzed by GC. Two saturated monoepoxy FAMEs, cis-9,10- and trans-9,10-epoxystearate, and four monounsaturated monoepoxy FAMEs, referred as monoepoxyoleates, cis-9,10-, trans-9,10-, cis-12,13-, and trans-12,13-epoxyoleate, were identified and quantitated in 
triolein and trilinolein, respectively. Results showed that such monoepoxy FAMEs constituted a major fraction within the group analyzed as oxidized FAME monomers, which account for the fatty acyl chains bearing any oxygen-containing functional group in TAGs (11).

In oils, owing to the different fatty acids involved in TAG and the presence of the minor compounds, specific research was necessary for accurate and sensitive quantitation of the monoepoxy FAMEs. Analytical solutions to eliminate interferences and to concentrate the compounds of interest were proposed. The methodology described was concluded to be very useful for the quantitation of the monoepoxy FAMEs in thermoxidized oils, as well as in refined oils in which they are present at levels as low as parts per million (12).

The objective of this study was to investigate the formation of monoepoxides in olive and sunflower oils subjected to frying temperature for different periods of time and to determine their levels in used frying oils sampled by the Food Inspection Services in restaurants and fried-food outlets.

\section{MATERIALS AND METHODS}

Chemicals. Methyl tridecanoate (C13:0) and methyl pentadecanoate (C15:0) were purchased from Nu-Check-Prep (Elysian, MN). Methyl cis-9,10-epoxystearate and $N$-methyl- $N$-nitroso- $p$-toluenesulfonamide were purchased from Sigma-Aldrich Chemie GmbH (Steinheim, Germany). Silica gel 60 for column chromatography (particle size $=$ $0.063-0.100 \mathrm{~mm}$ ) was acquired from Merck (Darmstadt, Germany). All other chemicals and reagents were of analytical grade and were obtained from local suppliers.

Samples. Olive oil (OO) and conventional sunflower oil (SO) were purchased from local outlets and thermoxidized at $180^{\circ} \mathrm{C}$ as described below. Used frying oils were sampled by the Food Inspection Services in restaurants and fried-food outlets in Andalusia (Spain) and used as received.

Heating Procedure. OO and SO samples $(2 \pm 0.01 \mathrm{~g})$ were weighed into standard glass tubes $(20 \mathrm{~cm} \times 12 \mathrm{~mm}$ i.d.). The tubes were introduced into Rancimat tubes, containing $8 \mathrm{~g}$ of glycerol to facilitate the heat transfer, and these were inserted in the heating block of the apparatus previously heated at $180 \pm 1{ }^{\circ} \mathrm{C}$. The tubes were left open to air during heating (13). Samples were heated for 5, 10, and $15 \mathrm{~h}$ and kept at $-20{ }^{\circ} \mathrm{C}$ until analyses.

Analysis of Monoepoxy Fatty Acids. Monoepoxides were analyzed by gas chromatography (GC) after derivatization to FAMEs. Quantitation was carried out using a mixture of methyl tridecanoate (C13:0) and methyl pentadecanoate (C15:0) as internal standards. Details on the procedure used were published elsewhere (12).

Derivatization Procedure. FAMEs were obtained in two consecutive steps, as described below: first, base-catalyzed transmethylation with sodium methoxide in tert-butyl methyl ether (TBME) at room temperature and, second, direct methylation of carboxylic groups with diazomethane.

A standard solution was prepared with a mixture of C13:0 (500 $\mu \mathrm{g} /$ $\mathrm{mL})$ and $\mathrm{C} 15: 0(500 \mu \mathrm{g} / \mathrm{mL})$ in TBME.

Transmethylation with Sodium Methoxide at Room Temperature. Samples of $100 \mathrm{mg}$ were accurately weighed into screw-capped centrifuge tubes, and $1 \mathrm{~mL}$ of the standard solution was added. Then, a $0.5-\mathrm{mL}$ volume of $0.2 \mathrm{M} \mathrm{NaOMe}$ solution in methanol was added, and the vial was closed, shaken for $1 \mathrm{~min}$, and left to rest at room temperature for $2 \mathrm{~min}$. For neutralization purposes, a $0.1-\mathrm{mL}$ volume of $0.5 \mathrm{M} \mathrm{H}_{2} \mathrm{SO}_{4}$ solution was added and the mixture was shaken for a few seconds. Then, $1.5 \mathrm{~mL}$ of water was added, shaken for $10 \mathrm{~s}$, and centrifuged. The organic layer was separated, evaporated to dryness under nitrogen, and redissolved in $2 \mathrm{~mL}$ of diethyl ether (14).

Methylation with Diazomethane. Diazomethane was prepared by the action of sodium hydroxide on $N$-methyl- $N$-nitroso- $p$-toluenesulfonamide using a device consisting of two connected tubes (15). The first tube was half-filled with diethyl ether $(\sim 5 \mathrm{~mL})$, and the second one contained $3 \mathrm{~mL}$ of 2-(2-ethoxyethoxy)ethanol, $3 \mathrm{~mL}$ of diethyl ether, and $3 \mathrm{~mL}$ of $60 \% \mathrm{NaOH}$ solution. Approximately $1 \mathrm{~g}$ of $N$-methyl$N$-nitroso- $p$-toluenesulfonamide was added into the second tube. A stream of nitrogen $(5-10 \mathrm{~mL} / \mathrm{min})$, saturated in ether vapor as passing through the first tube, was used to carry diazomethane generated in the second tube up to the vial containing the transmethylated sample diluted in diethyl ether. Methylation was considered to be complete when the sample turned yellow.

Gas-Liquid Chromatography (GLC). Monoepoxy FAMEs were analyzed by GLC using an HP 6890 series chromatograph (HewlettPackard, Avondale, PA). Samples $(\sim 50 \mathrm{mg} / \mathrm{mL})$ were injected into a split-splitless inlet operating in mode split with a 40:1 split ratio at $250{ }^{\circ} \mathrm{C}$. Injections $(2 \mu \mathrm{L})$ were carried out automatically using a 6890 series injector (Agilent Technologies, Karlsruhe, Germany). An HP Innowax fused-silica capillary column, $30 \mathrm{~m} \times 0.25 \mathrm{~mm}$ i.d., film thickness $=0.25 \mathrm{~mm}$, was used. Analyses were run using hydrogen ( 1 $\mathrm{mL} / \mathrm{min}$ ) as carrier gas and with the following temperature program: $90{ }^{\circ} \mathrm{C}$ held for $2 \mathrm{~min}$, raised at $4{ }^{\circ} \mathrm{C} / \mathrm{min}$ to $240{ }^{\circ} \mathrm{C}$, held for $20 \mathrm{~min}$. A flame ionization detector was used at $250{ }^{\circ} \mathrm{C}$ with hydrogen at 40 $\mathrm{mL} / \mathrm{min}$ and air at $450 \mathrm{~mL} / \mathrm{min}$ and nitrogen at $45 \mathrm{~mL} / \mathrm{min}$ as auxiliary gas.

Quantitative data for monoepoxy FAMEs were obtained by applying 0.97 as response factor (RF). This RF, which is referred to methyl pentadecanoate, was obtained for methyl cis-9,10-epoxystearate and used for each monoepoxy FAME, as previously reported (12).

Fatty Acid Composition. Fatty acid composition was performed by $\mathrm{GC}$ after derivatization to FAMEs with $2 \mathrm{~N} \mathrm{KOH}$ in methanol, according to the IUPAC Standard Method $(16,17)$.

Quantitation of Nonpolar and Polar FAMEs by Adsorption and Size Exclusion Chromatography. FAMEs from $300 \mathrm{mg}$ of oil were prepared by base-catalyzed transmethylation with sodium methoxide in TBME, as mentioned above. After evaporation of TBME under nitrogen, FAMEs were dissolved in $2 \mathrm{~mL}$ of $n$-hexane/diethyl ether $(95: 5, \mathrm{v} / \mathrm{v})$ and then separated into two fractions of different polarities by silica column chromatography. A 40-cm length and 1-cm i.d. glass column filled with $6 \mathrm{~g}$ of silica adjusted to a water content of $5 \%(\mathrm{~m} /$ $\mathrm{m})$ was used. The nonpolar fraction, which contains the unaltered FAMEs, was first eluted with $50 \mathrm{~mL}$ of $n$-hexane/diethyl ether (95:5, $\mathrm{v} / \mathrm{v})$. The polar fraction was subsequently eluted with $50 \mathrm{~mL}$ of diethyl ether. Such a fraction comprises the altered FAMEs including FAME polymers (FAP), FAME dimers (FAD), and oxidized FAME monomers (OxFAM). After evaporation of solvents, nonpolar and polar FAMEs were determined gravimetrically. The efficiency of the separation was assessed by thin-layer chromatography (TLC) using hexane/diethyl ether/acetic acid (80:20:1, v/v/v) for development of plates and exposure to iodine vapor to reveal the spots. The polar fraction obtained was dissolved in tetrahydrofuran (THF) at a concentration of $20-25 \mathrm{mg} /$ $\mathrm{mL}$. Quantitation of OxFAM, FAD, and FAP was performed by highperformance size exclusion chromatography (HPSEC). A Waters 510 pump (Waters Associates, Milford, MA) with a 10-mL sample loop was used. A Merck L-1490 refractive index detector and two 100- and 500- $\AA$ Ultrastyragel columns (Waters Associates) connected in series were used at room temperature. HPLC grade THF served as the mobile phase with a flow of $1 \mathrm{~mL} / \mathrm{min}$. This methodology was described in detail, including calibration and reproducibility data, in an earlier publication (18).

Statistical Analysis. Analytical determinations were carried out in triplicate unless otherwise indicated. Statistical analysis was performed by using Statistics software version 5.1 (StatSoft, Inc., Tulsa, OK). Statistical comparisons between means were made by applying Student's $t$ test. Significance was defined at $p<0.05$.

\section{RESULTS AND DISCUSSION}

Table 1 shows results of major fatty acids found in initial samples of $\mathrm{OO}$ and $\mathrm{SO}$ and samples heated at $180{ }^{\circ} \mathrm{C}$ for 5,10 , and $15 \mathrm{~h}$. Data of nonpolar FAME fractions determined by adsorption chromatography have also been included. This fraction accounts for the fatty acyl chains in TAGs, which remain unaltered during heating. As can be observed in fatty acid composition, the level of saturated fatty acids did not 
Table 1. Nonpolar FAME Content and Fatty Acid Composition of Olive Oil and Sunflower Oil, Samples Nonheated and Heated at $180{ }^{\circ} \mathrm{C}$ for 5 , 10 , and $15 \mathrm{~h}$

\begin{tabular}{|c|c|c|c|c|c|c|}
\hline \multirow[b]{2}{*}{ sample } & \multirow{2}{*}{$\begin{array}{l}\text { nonpolar FAME }{ }^{a} \\
\text { (wt } \% \text { on oil) }\end{array}$} & \multicolumn{5}{|c|}{ fatty acid composition (wt \% on oil) } \\
\hline & & $\mathrm{C} 16: 0$ & C16:1 & C18:0 & $\mathrm{C} 18: 1$ & C18:2 \\
\hline $\begin{array}{l}00,0 \mathrm{~h}^{b} \\
00,5 \mathrm{~h} \\
00,10 \mathrm{~h} \\
00,15 \mathrm{~h}\end{array}$ & $\begin{array}{l}98.6 \pm 0.25^{d} \\
94.7 \pm 0.26 \\
90.1 \pm 0.26 \\
84.5 \pm 0.42\end{array}$ & $\begin{array}{l}11.3 \pm 0.10 \\
11.1 \pm 0.14 \\
10.9 \pm 0.17 \\
10.6 \pm 0.15\end{array}$ & $\begin{array}{l}0.9 \pm 0.06 \\
0.9 \pm 0.14 \\
0.8 \pm 0.06 \\
0.8 \pm 0.06\end{array}$ & $\begin{array}{l}2.7 \pm 0.06 \\
2.7 \pm 0.06 \\
2.5 \pm 0.10 \\
2.6 \pm 0.12\end{array}$ & $\begin{array}{l}76.0 \pm 0.20 \\
73.4 \pm 0.26 \\
69.8 \pm 0.12 \\
66.5 \pm 0.25\end{array}$ & $\begin{array}{l}7.7 \pm 0.06 \\
6.4 \pm 0.10 \\
5.3 \pm 0.12 \\
4.4 \pm 0.12\end{array}$ \\
\hline $\begin{array}{l}\text { SO, } 0 \mathrm{~h}^{\mathrm{c}} \\
\text { SO, } 5 \mathrm{~h} \\
\text { SO, } 10 \mathrm{~h} \\
\text { SO, } 15 \mathrm{~h}\end{array}$ & $\begin{array}{l}99.4 \pm 0.29 \\
93.3 \pm 0.55 \\
87.9 \pm 0.15 \\
82.2 \pm 0.15\end{array}$ & $\begin{array}{l}6.2 \pm 0.10 \\
6.5 \pm 0.15 \\
6.1 \pm 0.17 \\
6.2 \pm 0.12\end{array}$ & $\begin{array}{l}\operatorname{tr} e \\
\operatorname{tr} \\
\operatorname{tr} \\
\operatorname{tr}\end{array}$ & $\begin{array}{l}4.7 \pm 0.06 \\
4.7 \pm 0.14 \\
4.6 \pm 0.06 \\
4.5 \pm 0.06\end{array}$ & $\begin{array}{l}27.7 \pm 0.12 \\
26.6 \pm 0.35 \\
25.3 \pm 0.21 \\
24.5 \pm 0.36\end{array}$ & $\begin{array}{l}60.8 \pm 0.25 \\
55.9 \pm 0.36 \\
51.9 \pm 0.31 \\
47.6 \pm 0.35\end{array}$ \\
\hline
\end{tabular}

${ }^{a}$ Nonpolar fatty acid methyl esters. ${ }^{b}$ Olive oil and heating time. ${ }^{c}$ Sunflower oil and heating time. ${ }^{d}$ Mean \pm standard deviation $(n=3) .{ }^{e}$ Traces $<0.05 \%$.

Table 2. Polar FAME Content and Distribution in Fatty Acid Polymers, Dimers, and Oxidized Monomers in Olive Oil and Sunflower Oil Heated at $180{ }^{\circ} \mathrm{C}$ for 5,10 , and $15 \mathrm{~h}$

\begin{tabular}{ccccc}
\hline & & \multicolumn{3}{c}{ distribution (wt \% on oil) } \\
\cline { 3 - 5 } sample & (wt \% on oil) & FAPb & FAD $^{c}$ & OxFAM $^{d}$ \\
\hline OO, $5 \mathrm{~h}^{\mathrm{e}}$ & $5.4 \pm 0.21^{g}$ & $0.7 \pm 0.11$ & $1.9 \pm 0.14$ & $2.9 \pm 0.06$ \\
OO, 10 h & $9.9 \pm 0.28$ & $1.4 \pm 0.23$ & $3.6 \pm 0.47$ & $4.9 \pm 0.40$ \\
OO, $15 \mathrm{~h}$ & $15.5 \pm 0.28$ & $3.0 \pm 0.35$ & $6.1 \pm 0.25$ & $6.4 \pm 0.34$ \\
SO, $5 \mathrm{~h}^{\dagger}$ & $6.5 \pm 0.42$ & $0.9 \pm 0.06$ & $3.1 \pm 0.26$ & $2.5 \pm 0.16$ \\
SO $10 \mathrm{~h}$ & $12.2 \pm 0.07$ & $1.1 \pm 0.09$ & $6.1 \pm 0.16$ & $4.9 \pm 0.26$ \\
SO, 15 h & $17.5 \pm 0.28$ & $3.7 \pm 0.27$ & $8.7 \pm 0.18$ & $5.2 \pm 0.40$
\end{tabular}

${ }^{a}$ Polar fatty acid methyl esters. ${ }^{b}$ Fatty acid methyl ester polymers. ${ }^{c}$ Fatty acid methyl ester dimers. ${ }^{d}$ Oxidized fatty acid methyl ester monomers. ${ }^{e}$ Olive oil and heating time. ${ }^{f}$ Sunflower oil and heating time. ${ }^{g}$ Mean \pm standard deviation $(n=$ 3).

change significantly, whereas that of unsaturated fatty acids decreased, as expected, with the heating time. Losses of both major fatty acids, that is, oleic and linoleic acids, were even detected after $5 \mathrm{~h}$ of heating. The amounts of nonpolar FAMEs were of the same order for both oils after each period of heating, although statistical analysis showed significantly lower values for the SO samples.

Table 2 shows results of total polar FAME fractions, accounting for the altered fatty acyl chains in TAGs, and its distribution in FAP, FAD, and OxFAM, formed through thermal and oxidative reactions during heating. The total amount of polar FAMEs was slightly higher for SO than for OO after each period of heating. Different distributions of polar FAMEs were found between $\mathrm{OO}$ and SO. It can be observed that, for similar degradation levels, OO showed lower amounts of polymers (including FAP and FAD), indicating a lower tendency to polymerization in comparison to SO. These results are in agreement with previous results showing a lower tendency to polymerization of monounsaturated oils as compared to polyunsaturated oils (19).

Quantitation of six monoepoxy FAMEs, previously identified in thermoxidized oils (12), was obtained by analyzing total FAMEs. No interference due to the nonpolar FAME fraction was observed. In a previous work, it was shown that behenic acid methyl ester (C22:0) eluted overlapping with the compounds of interest, and analysis of the polar FAME fraction was proposed (12). However, as illustrated in Figure 1, showing the significant part of GC chromatograms of OO and SO heated for 5 and $10 \mathrm{~h}$, respectively, the chromatographic conditions applied in the present study allowed the separation of C22:0.

Quantitative results for monoepoxy FAMEs found for thermoxidized $\mathrm{OO}$ and $\mathrm{SO}$ are listed in Table 3. The total content

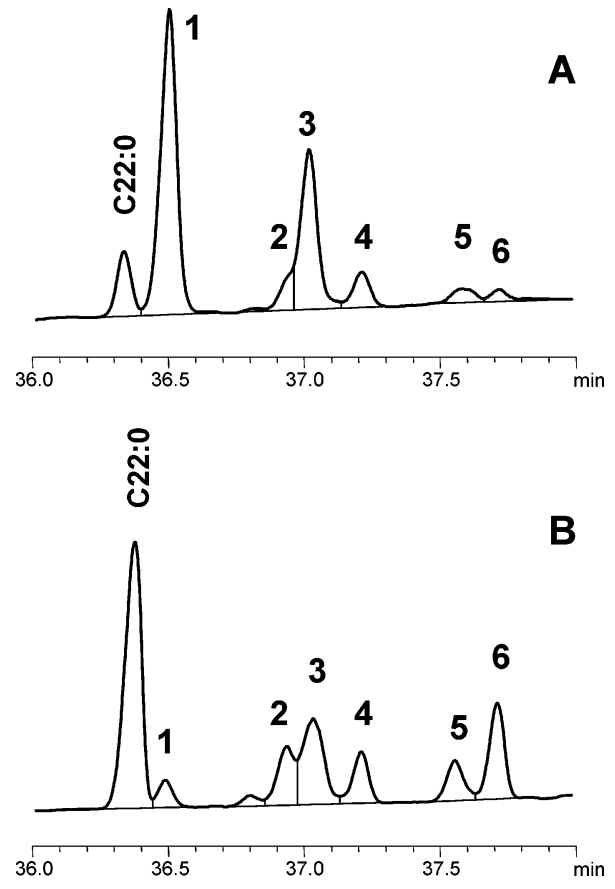

Figure 1. Significant part of the gas chromatograms corresponding to the zone of monoepoxy FAMEs of olive oil (A) and sunflower oil (B) heated at $180{ }^{\circ} \mathrm{C}$ for 5 and $10 \mathrm{~h}$, respectively. Peaks: C22:0, behenic acid methyl ester; 1, methyl trans-9,10-epoxystearate; 2, methyl trans12,13-epoxyoleate; 3, methyl cis-9,10-epoxystearate; 4, methyl trans-9,10-epoxyoleate; 5, methyl cis-12,13-epoxyoleate; 6, methyl cis-9,10epoxyoleate.

of the monoepoxy FAMEs quantitated in this study ranged from 4.29 to $14.24 \mathrm{mg} / \mathrm{g}$ of oil in $\mathrm{OO}$ and from 5.10 to $9.44 \mathrm{mg} / \mathrm{g}$ of oil in SO for the periods of heating assayed. These amounts constituted major fractions approximately of the same order within the group of OxFAM of both oils (Table 2). Thus, monoepoxy FAMEs constituted $14.8-22.3 \%$ of OxFAM for OO and $12.4-20.4 \%$ for SO. On the total polar FAME fraction, monoepoxy FAMEs ranged from 7.4 to $9.2 \%$ for $\mathrm{OO}$ and from 5.0 to $7.8 \%$ for SO. These contents were relatively high considering that monoepoxy FAMEs make up only six specific compounds among the large number of new compounds formed during thermoxidation.

No statistical differences in the total content of monoepoxy FAMEs were found between $\mathrm{OO}$ and $\mathrm{SO}$ for the samples heated for 5 and $10 \mathrm{~h}$. Nevertheless, OO showed significantly higher contents of total monoepoxy FAMEs than SO for the samples heated for $15 \mathrm{~h}$, indicating a more rapid evolution of the compounds of interest in OO. As will be discussed below, this 
Table 3. Monoepoxy FAME Content (Milligrams per Gram of Oil) in Olive Oil and Sunflower Oil Heated at $180{ }^{\circ} \mathrm{C}$ for 5,10 , and $15 \mathrm{~h}$

\begin{tabular}{|c|c|c|c|c|c|c|}
\hline \multirow[b]{2}{*}{ monoepoxide ${ }^{a}$} & \multicolumn{3}{|c|}{ olive oil } & \multicolumn{3}{|c|}{ sunflower oil } \\
\hline & $5 \mathrm{~h}$ & $10 \mathrm{~h}$ & $15 \mathrm{~h}$ & $5 \mathrm{~h}$ & $10 \mathrm{~h}$ & $15 \mathrm{~h}$ \\
\hline cis-9,10-ES & $1.30 \pm 0.199^{b}$ & $2.14 \pm 0.009$ & $4.14 \pm 0.017$ & $1.48 \pm 0.052$ & $1.64 \pm 0.165$ & $1.96 \pm 0.010$ \\
\hline trans-9,10-ES & $2.10 \pm 0.069$ & $3.91 \pm 0.191$ & $7.61 \pm 0.035$ & $0.23 \pm 0.017$ & $0.34 \pm 0.017$ & $0.84 \pm 0.026$ \\
\hline cis-9,10-EO & $0.15 \pm 0.036$ & $0.18 \pm 0.026$ & $0.43 \pm 0.110$ & $1.51 \pm 0.245$ & $1.53 \pm 0.305$ & $2.15 \pm 0.121$ \\
\hline trans-9,10-EO & $0.28 \pm 0.017$ & $0.44 \pm 0.017$ & $0.86 \pm 0.078$ & $0.49 \pm 0.087$ & $0.80 \pm 0.162$ & $1.45 \pm 0.087$ \\
\hline cis-12,13-EO & $0.16 \pm 0.017$ & $0.25 \pm 0.139$ & $0.30 \pm 0.061$ & $0.51 \pm 0.022$ & $0.68 \pm 0.103$ & $1.18 \pm 0.174$ \\
\hline trans-12,13-EO & $0.30 \pm 0.020$ & $0.40 \pm 0.017$ & $0.90 \pm 0.075$ & $0.88 \pm 0.132$ & $1.10 \pm 0.174$ & $1.86 \pm 0.010$ \\
\hline total & $4.29 \pm 0.303$ & $7.32 \pm 0.346$ & $14.24 \pm 0.308$ & $5.10 \pm 0.482$ & $6.09 \pm 0.685$ & $9.44 \pm 0.217$ \\
\hline
\end{tabular}

${ }^{a}$ ES, epoxystearate; EO, epoxyoleate. ${ }^{b}$ Mean \pm standard deviation $(n=3)$.
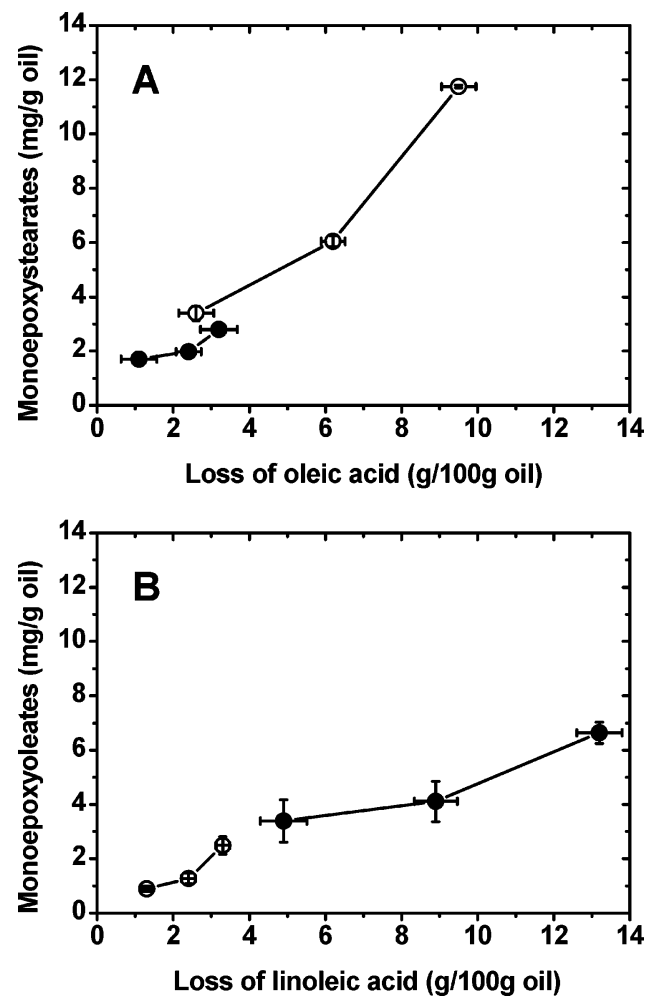

Figure 2. Relationships between contents of monoepoxystearates and corresponding losses of oleic acid $(\mathbf{A})$ and between contents of monoepoxyoleates and corresponding losses of linoleic acid $(\mathbf{B})$ in samples of olive oil $(\bigcirc)$ and sunflower oil $(\bullet)$ heated at $180^{\circ} \mathrm{C}$ for 5 , 10 , and 15 h. Error bars denote standard deviations $(n=3)$.

fact is in part related to the lower tendency to polymerization shown by $\mathrm{OO}$ as compared to SO.

Formation of monoepoxystearates and monoepoxyoleates was observed for both oils after each period of heating. This fact was expected from the data of fatty acid composition in Table 1, which show degradation of both oleic and linoleic acids in all of the samples. Moreover, the major monoepoxy FAMEs found for $\mathrm{OO}$ and $\mathrm{SO}$ corresponded to degradation of the major unsaturated fatty acid of each oil. Thus, on the total monoepoxy FAMEs, monoepoxystearates, which arise from oleic acid, ranged from 79.3 to $82.6 \%$ in $\mathrm{OO}$ and from 29.7 to $33.5 \%$ in $\mathrm{SO}$, whereas monoepoxyoleates, arising from linoleic acid, ranged from 17.4 to $20.7 \%$ in $\mathrm{OO}$ and from 66.5 to $70.3 \%$ in SO. In this respect, it is interesting to examine the relationship between the formation of monoepoxides and losses of their corresponding fatty acids. Figure 2 shows amounts of monoepoxystearates in both oils against corresponding losses of oleic acid (A) and amounts of monoepoxyoleates against losses of linoleic acid (B). As can be observed, for the same amount of fatty acid loss, the amounts of monoepoxystearates found in
OO were significantly higher than those of monoepoxyoleates in SO. These results can be in part attributed to the different tendency to polymerization shown by both oils. From the results in Tables $\mathbf{1}$ and $\mathbf{2}$ it is inferred that the major fatty acid in SO, that is, linoleic acid, had a higher participation in the formation of polymerization compounds (FAD + FAP) than did oleic acid, the major fatty acid in OO. Therefore, higher losses of linoleic acid in SO than those of oleic acid in $\mathrm{OO}$ can be expected for the same amount of the corresponding monoepoxides. In addition to this different tendency to formation of the major monoepoxides of each oil, different stabilities are expected for saturated and monounsaturated monoepoxides. Whereas monoepoxyoleates may participate through the double bond in further secondary reactions, monoepoxystearates would accumulate due to the absence of double bonds.

No consistent patterns were found for OO and SO concerning the cis/trans isomery of the epoxy ring. As can be observed in Table 3, the amounts of trans isomers were with no exception higher than those of their cis isomers counterparts in the OO samples. In contrast, the SO samples showed significantly lower amounts of trans than cis isomers for the epoxy ring at the 9-10-position, and this was commonly observed for epoxyoleates and epoxystearates.

Formation of the epoxy ring at the 9-10-position of linoleic acid seemed to be favored in the SO samples, as higher amounts of 9,10-epoxyoleates than of 12,13-epoxyoleates were found. For the OO samples, the increase of monoepoxides arising from linoleic acid was so low that this tendency was not observed.

Table 4 lists results of fatty acid composition and nonpolar FAME fractions of used frying oils. The samples have been ordered by increasing levels of degree of unsaturation. Thus, three groups can be differentiated by means of the oleic-tolinoleic acid ratio, which ranged from 10.7 to 19.3 in samples $1-3$, from 4.3 to 5.0 in samples 4 and 5 , and from 0.5 to 1.0 in samples 6-10. Within each subgroup, samples have been numbered by decreasing contents of the nonpolar FAME fraction.

Contents of polar FAMEs, indicating the level of degradation, and their distribution in the main groups of new compounds are given in Table 5. Very different degradation levels can be observed among the frying oils, which expressed in terms of polar FAMEs ranged from 9.0 to $28.2 \%$. It is interesting to emphasize that even in these samples of variable levels of degradation and unknown history, the lower tendency to polymerization shown by monounsaturated oils (samples $1-5$ ) as compared to polyunsaturated oils (samples 6-10) was observed again. This different tendency can be expressed in terms of the polymers-to-oxidized monomers ratio (i.e., the sum of FAD and FAP-to-OxFAM ratio), which ranged from 0.91 to 1.29 in samples $1-5$ and between 1.50 and 3.17 in samples $6-10$. 
Table 4. Nonpolar FAME Content and Fatty Acid Composition of Used Frying Oils from Restaurants and Fried-Food Outlets in Spain

\begin{tabular}{|c|c|c|c|c|c|c|c|}
\hline \multirow[b]{2}{*}{ sample } & \multirow{2}{*}{$\begin{array}{l}\text { nonpolar FAME }{ }^{a} \\
\text { (wt } \% \text { on oill) }\end{array}$} & \multicolumn{6}{|c|}{ fatty acid composition (wt \% on oil) } \\
\hline & & $\mathrm{C} 16: 0$ & C16:1 & C18:0 & C18:1 & C18:2 & others \\
\hline 1 & $87.3^{b}$ & $8.6 \pm 0.03^{c}$ & $0.6 \pm 0.09$ & $2.9 \pm 0.09$ & $67.8 \pm 0.08$ & $4.0 \pm 0.08$ & $3.13 \pm 0.20$ \\
\hline 2 & 87.0 & $10.9 \pm 0.12$ & $0.9 \pm 0.11$ & $3.3 \pm 0.26$ & $62.3 \pm 1.13$ & $5.8 \pm 0.20$ & $3.63 \pm 1.07$ \\
\hline 3 & 81.2 & $8.7+0.10$ & $0.7+0.12$ & $2.7+0.04$ & $61.6+0.07$ & $3.2 \pm 0.22$ & $4.29+0.13$ \\
\hline 4 & 87.0 & $7.2 \pm 0.06$ & $0.5 \pm 0.05$ & $4.0 \pm 0.21$ & $58.5 \pm 0.06$ & $13.5 \pm 0.23$ & $3.28 \pm 0.09$ \\
\hline 5 & 86.9 & $5.0 \pm 0.09$ & $0.3 \pm 0.05$ & $3.8 \pm 0.31$ & $62.4 \pm 1.28$ & $12.4 \pm 0.25$ & $2.94 \pm 1.18$ \\
\hline 6 & 91.0 & $6.7 \pm 0.07$ & $0.2 \pm 0.05$ & $4.1 \pm 0.13$ & $28.6 \pm 0.22$ & $49.4 \pm 0.07$ & $2.02 \pm 0.15$ \\
\hline 7 & 89.8 & $7.6 \pm 0.02$ & $0.4 \pm 0.03$ & $3.9 \pm 0.02$ & $37.9 \pm 0.10$ & $38.2 \pm 0.15$ & $1.82 \pm 0.28$ \\
\hline 8 & 89.0 & $9.0 \pm 0.08$ & $0.7 \pm 0.01$ & $3.4 \pm 0.03$ & $26.6 \pm 0.21$ & $47.4 \pm 0.15$ & $1.92 \pm 0.01$ \\
\hline 9 & 82.9 & $7.9 \pm 0.02$ & $0.3 \pm 0.10$ & $4.7 \pm 0.28$ & $29.7 \pm 0.56$ & $33.7 \pm 0.46$ & $6.54 \pm 0.85$ \\
\hline 10 & 71.8 & $5.4 \pm 0.01$ & $0.2 \pm 0.02$ & $3.9 \pm 0.13$ & $19.0 \pm 0.14$ & $40.3 \pm 0.14$ & $3.05 \pm 0.19$ \\
\hline
\end{tabular}

${ }^{a}$ Nonpolar fatty acid methyl esters. ${ }^{b}$ Mean $(n=2) .{ }^{c}$ Mean \pm standard deviation $(n=3)$.

Table 5. Polar FAME Content and Distribution in Fatty Acid Polymers, Dimers, and Oxidized Monomers in Used Frying Oils from Restaurants and Fried-Food Outlets in Spain

\begin{tabular}{ccccc}
\hline & & \multicolumn{3}{c}{ distribution (wt \% on oil) } \\
\cline { 3 - 5 } sample & $\begin{array}{c}\text { polar FAME } \\
\text { (wt \% on oil) }\end{array}$ & FAP $^{b}$ & FAD $^{c}$ & OxFAM $^{d}$ \\
\hline 1 & $12.7^{e}$ & 3.1 & 3.7 & 5.9 \\
2 & 13.0 & 1.5 & 4.7 & 6.8 \\
3 & 18.8 & 4.7 & 5.9 & 8.2 \\
4 & 13.0 & 1.6 & 5.3 & 6.0 \\
5 & 13.1 & 1.7 & 4.6 & 6.8 \\
6 & 9.0 & 2.2 & 3.2 & 3.6 \\
7 & 10.2 & 2.5 & 4.0 & 3.7 \\
8 & 11.0 & 1.4 & 5.3 & 4.3 \\
9 & 17.1 & 4.3 & 8.7 & 4.1 \\
10 & 28.2 & 9.1 & 11.6 & 7.6
\end{tabular}

${ }^{a}$ Polar fatty acid methyl esters. ${ }^{b}$ Fatty acid methyl ester polymers. ${ }^{c}$ Fatty acid methyl ester dimers. ${ }^{d}$ Oxidized fatty acid methyl ester monomers. ${ }^{e}$ Mean $(n=$ 2).

Quantitative results of monoepoxy FAMEs in used frying oils are shown in Table 6. The total amount of the monoepoxy FAMEs ranged from 12.29 to $14.42 \mathrm{mg} / \mathrm{g}$ pf oil in samples $1-3$, from 4.83 to $5.38 \mathrm{mg} / \mathrm{g}$ of oil in samples 4 and 5 , and from 3.37 to $11.74 \mathrm{mg} / \mathrm{g}$ of oil in samples 6-10. Results in Tables 5 and $\mathbf{6}$ show that, for similar degradation levels, the total content of monoepoxy FAMEs was higher for the samples of lower degree of unsaturation. As previously discussed for the thermoxidized oils, this fact is attributable to two concurrent reasons: lower tendency to polymerization and enhanced formation of stable monoepoxides, that is, monoepoxystearates. In this respect, it was observed that monoepoxystearates increased exponentially as the oleic-to-linoleic acid ratio was higher (Figure 3).

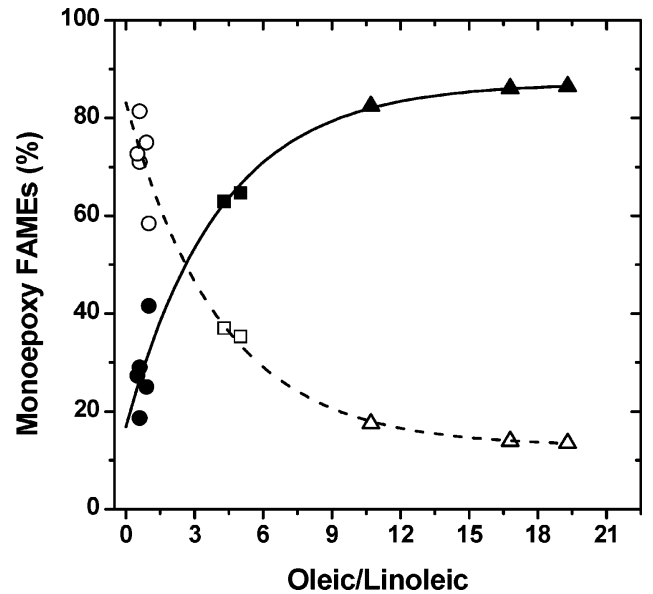

Figure 3. Relative contents of monoepoxystearates (solid symbols) and monoepoxyoleates (open symbols) against the oleic-to-linoleic acid ratio for used frying oils. Triangles represent values for samples $1-3$, squares those for samples 4 and 5, and circles those for samples 6-10.

It is important to stress the great differences in the total content of monoepoxides found among the monounsaturated oils as a function of the content of linoleic acid, the most susceptible to degradation in the oils studied. This fact is clearly reflected if samples 1-3 and samples 4 and 5 are compared.

As previously found for thermoxidized OO, higher contents of the trans epoxides than their cis counterparts were found for the monounsaturated oils (samples 1-5). With the exception of the most altered sample (sample 10), the polyunsaturated oils showed, as also previously found for thermoxidized SO, lower contents of trans isomers than cis for the epoxy ring at the 9-10-position. In contrast, for sample 10 the contents of trans isomers were with no exceptions higher than those of corre-

Table 6. Monoepoxy FAME Content (Milligrams per Gram of Oil) in Used Frying Oils from Restaurants and Fried-Food Outlets in Spain

\begin{tabular}{|c|c|c|c|c|c|c|c|}
\hline \multirow[b]{2}{*}{ sample } & \multicolumn{2}{|c|}{ epoxystearates } & \multicolumn{4}{|c|}{ epoxyoleates } & \multirow[b]{2}{*}{ total } \\
\hline & cis-9,10- & trans-9,10- & cis-9,10- & trans-9,10- & cis-12,13- & trans-12,13- & \\
\hline 1 & $5.63 \pm 0.112^{a}$ & $6.79 \pm 0.035$ & $0.30 \pm 0.010$ & $0.62 \pm 0.035$ & $0.34 \pm 0.017$ & $0.75 \pm 0.035$ & $14.42 \pm 0.121$ \\
\hline 2 & $4.62 \pm 0.009$ & $5.53 \pm 0.017$ & $0.31 \pm 0.006$ & $0.69 \pm 0.017$ & $0.38 \pm 0.017$ & $0.77 \pm 0.026$ & $12.29 \pm 0.035$ \\
\hline 3 & $4.73 \pm 0.013$ & $7.60 \pm 0.112$ & $0.21 \pm 0.026$ & $0.73 \pm 0.017$ & $0.33 \pm 0.006$ & $0.65 \pm 0.087$ & $14.24 \pm 0.026$ \\
\hline 4 & $1.44 \pm 0.052$ & $1.96 \pm 0.017$ & $0.39 \pm 0.026$ & $0.51 \pm 0.009$ & $0.28 \pm 0.009$ & $0.82 \pm 0.035$ & $5.38 \pm 0.087$ \\
\hline 5 & $1.53 \pm 0.009$ & $1.61 \pm 0.043$ & $0.26 \pm 0.017$ & $0.56 \pm 0.006$ & $0.26 \pm 0.013$ & $0.63 \pm 0.061$ & $4.83 \pm 0.043$ \\
\hline 6 & $0.42 \pm 0.078$ & $0.21 \pm 0.060$ & $0.83 \pm 0.035$ & $0.40 \pm 0.035$ & $0.37 \pm 0.009$ & $1.15 \pm 0.087$ & $3.37 \pm 0.025$ \\
\hline 7 & $1.49 \pm 0.303$ & $0.48 \pm 0.038$ & $0.68 \pm 0.128$ & $0.56 \pm 0.087$ & $0.56 \pm 0.069$ & $0.97 \pm 0.199$ & $4.72 \pm 0.510$ \\
\hline 8 & $0.84 \pm 0.072$ & $0.31 \pm 0.072$ & $0.49 \pm 0.043$ & $0.64 \pm 0.069$ & $0.50 \pm 0.052$ & $1.19 \pm 0.011$ & $3.96 \pm 0.442$ \\
\hline 9 & $2.06 \pm 0.035$ & $0.61 \pm 0.026$ & $3.57 \pm 0.138$ & $1.46 \pm 0.078$ & $1.21 \pm 0.135$ & $1.75 \pm 0.026$ & $10.64 \pm 0.356$ \\
\hline 10 & $1.39 \pm 0.091$ & $1.82 \pm 0.199$ & $1.49 \pm 0.051$ & $2.46 \pm 0.017$ & $1.33 \pm 0.017$ & $3.26 \pm 0.014$ & $11.74 \pm 0.312$ \\
\hline
\end{tabular}

\footnotetext{
a Mean \pm standard deviation $(n=3)$.
} 
sponding cis, suggesting that formation of the thermodynamically more stable isomers (trans) may occur as degradation increases.

No significant differences were found between amounts of 9,10- and 12,13-epoxyoleates in the monounsaturated oils. However, and contrary to what was found in the thermoxidized SO samples, the polyunsaturated oils showed significantly higher contents of 12,13-epoxyoleates than 9,10-epoxyoleates, with the exception of sample 9 .

It is concluded that, for similar levels of degradation, monounsaturated oils are characterized by a higher content of monoepoxides than polyunsaturated oils. This fact is attributable to two concurrent reasons: first, a lower tendency to polymerization of monounsaturated oils, reflected by a lower polymersto-oxidized monomers ratio, and, second, greater stability and hence accumulation of the major monoepoxides formed in monounsaturated oils, that is, monoepoxystearates, in contrast to the susceptibility to further reactions of the most abundant monoepoxides found in polyunsaturated oils, that is, monoepoxyoleates.

Much work remains to be done to clarify the kinetics of formation and degradation of the monoepoxides at high temperature, of undoubted interest to justify the differences found between the contents of isomeric cis and trans forms.

\section{ACKNOWLEDGMENT}

We thank M. Giménez for assistance.

\section{LITERATURE CITED}

(1) Cohn, J. Oxidized fat in the diet, postprandial lipaemia and cardiovascular disease. Curr. Opin. Lipidol. 2002, 13, 19-24.

(2) Dobarganes, M. C.; Márquez-Ruiz, G. Oxidised fats in foods. Curr. Opin. Clin. Nutr. Metab. Care 2003, 6, 157-163.

(3) Márquez-Ruiz, G.; Martín-Polvillo, M.; Dobarganes, M. C. Quantitation of oxidized triglyceride monomers and dimers as a useful measurement for early and advanced stages of oxidation. Grasas Aceites 1996, 47, 48-53.

(4) Firestone, D. Regulation of frying fats and oils. In Deep Frying: Chemistry, Nutrition and Practical Applications; Perkins, E. G., Erickson, M. D., Eds.; American Oil Chemists' Society: Champaign, IL, 1996; pp 323-334.

(5) Saguy, I. S.; Dana, D. Integrated approach to deep fat frying: engineering, nutrition, health and consumer aspects. J. Food Eng. 2003, 56, 143-152.

(6) Dobarganes, M. C.; Pérez-Camino, M. C.; Márquez-Ruiz, G. High performance size exclusion chromatography of polar compounds in heated and non-heated fats. Fat Sci. Technol. 1988, 90, 308-311.
(7) Márquez-Ruiz, G.; Dobarganes, M. C. Nutritional and physiological effects of used frying fats. In Deep Frying: Chemistry, Nutrition and Practical Applications; Perkins, E. G., Erickson, M. D., Eds.; American Oil Chemists' Society: Champaign, IL, 1996; pp 160-182.

(8) Billek, G. Health aspects of thermoxidized oils and fats. Eur. J. Lipid Sci. Technol. 2000, 102, 587-593.

(9) Frankel, E. N. Hydroperoxide decomposition. In Lipid Oxidation; Frankel E. N., Ed.; The Oily Press: Dundee, U.K., 1998; pp 55-77.

(10) Wilson, R.; Fernie, C. E.; Scrimgeour, C. M.; Lyall, K.; Smyth, L.; Riemersma, R. A. Dietary epoxy fatty acids are absorbed in healthy women. Eur. J. Clin. Invest. 2002, 32, 79-83.

(11) Berdeaux, O.; Márquez-Ruiz, G.; Dobarganes, M. C. Characterization, quantitation and evolution of monoepoxy compounds formed in model systems of fatty acids methyl esters and monoacid triglycerides heated at high temperature. Grasas Aceites 1999, 50, 53-59.

(12) Velasco, J.; Berdeaux, O.; Márquez-Ruiz, G.; Dobarganes, M. C. Sensitive and accurate quantitation of monoepoxy fatty acids in thermoxidized oils by gas-liquid chromatography. J. Chromatogr. 2002, 982, 145-152.

(13) Barrera-Arellano, D.; Márquez-Ruiz, G.; Dobarganes, M. C. A simple procedure to evaluate the performance of fats and oils at frying temperatures. Grasas Aceites 1997, 48, 231-235.

(14) Cecchi, G.; Biasini, S.; Castano, J. Méthanolyse rapide des huiles en solvant. Rev. Fr. Corps Gras 1985, 32, 163-164.

(15) Cohen, J. D. Convenient Apparatus for the generation of small amounts of diazomethane. J. Chromatogr. 1984, 303, 193-196.

(16) IUPAC. Preparation of the fatty acid methyl esters 2.301. In Standard Methods for the Analysis of Oils, Fats and Derivatives, 7th ed.; International Union of Pure and Applied Chemistry, Blackwell Scientific: Oxford, U.K., 1992.

(17) IUPAC. Gas-liquid chromatography of fatty acid methyl esters 2.302. In Standard Methods for the Analysis of Oils, Fats and Derivatives, 7th ed.; International Union of Pure and Applied Chemistry, Blackwell Scientific: Oxford, U.K., 1992.

(18) Márquez-Ruiz, G.; Pérez-Camino, M. C.; Dobarganes, M. C. Combination of adsorption and size-exclusion chromatography for the determination of fatty acid monomers, dimers and polymers. J. Chromatogr. 1990, 514, 37-44.

(19) Márquez-Ruiz, G.; Tasioula-Margari, M.; Dobarganes, M. C. Quantitation and distribution of altered fatty acids in frying fats. J. Am. Oil Chem. Soc. 1995, 72, 1171-1176.

Received for review November 7, 2003. Revised manuscript received April 8, 2004. Accepted April 29, 2004. This work was funded by the Ministerio de Ciencia y Tecnología (Project ALI 2001-0505). O.B. was supported by a grant from the EU (Contract FAIR-97-5016).

JF030753F 